\title{
Disorder-Free Localization in an Interacting 2D Lattice Gauge Theory
}

\author{
P. Karpov $\odot,{ }^{1,2,}{ }^{*}$ R. Verdel๑, ${ }^{1}$ Y.-P. Huang, ${ }^{3,4}$ M. Schmitt $\odot,{ }^{5}$ and M. Heyl ${ }^{1}$ \\ ${ }^{1}$ Max Planck Institute for the Physics of Complex Systems, Nöthnitzer Straße 38, Dresden 01187, Germany \\ ${ }^{2}$ National University of Science and Technology “MISiS,” Moscow 119991, Russia \\ ${ }^{3}$ The Paul Scherrer Institute, Forschungsstrasse 111, 5232 Villigen, Switzerland \\ ${ }^{4}$ Department of Physics, National Tsing Hua University, Hsinchu 30013, Taiwan \\ ${ }^{5}$ Department of Physics, University of California at Berkeley, Berkeley, California 94720, USA
}

(Received 13 March 2020; revised 24 December 2020; accepted 4 February 2021; published 1 April 2021)

\begin{abstract}
Disorder-free localization has been recently introduced as a mechanism for ergodicity breaking in lowdimensional homogeneous lattice gauge theories caused by local constraints imposed by gauge invariance. We show that also genuinely interacting systems in two spatial dimensions can become nonergodic as a consequence of this mechanism. This result is all the more surprising since the conventional many-body localization is conjectured to be unstable in two dimensions; hence the gauge invariance represents an alternative robust localization mechanism surviving in higher dimensions in the presence of interactions. Specifically, we demonstrate nonergodic behavior in the quantum link model by obtaining a bound on the localization-delocalization transition through a classical correlated percolation problem implying a fragmentation of Hilbert space on the nonergodic side of the transition. We study the quantum dynamics in this system by introducing the method of "variational classical networks," an efficient and perturbatively controlled representation of the wave function in terms of a network of classical spins akin to artificial neural networks. We identify a distinguishing dynamical signature by studying the propagation of line defects, yielding different light cone structures in the localized and ergodic phases, respectively. The methods we introduce in this work can be applied to any lattice gauge theory with finite-dimensional local Hilbert spaces irrespective of spatial dimensionality.
\end{abstract}

DOI: $10.1103 /$ PhysRevLett.126.130401

Introduction.-Systems with local constraints play an important role in various physical contexts ranging from strongly correlated electrons $[1,2]$ and frustrated magnets [3-5] to quantum information [6] and fundamental theories of matter such as quantum electrodynamics and chromodynamics [7], where constraints take the form of local gauge symmetries. The equilibrium properties of such systems have been extensively studied over the past decades, but only recently their nonequilibrium dynamics has moved into focus. In particular, local constraints have emerged as a new paradigm for ergodicity breaking, besides the two known archetypical scenarios caused by localization due to strong disorder or integrability. Systems with local constraints can exhibit rare nonergodic eigenstates, termed quantum many-body scars $[8,9]$, or extremely slow relaxation [10-12], whereas dipole conservation can prevent thermalization of large parts of the spectrum in one-dimensional fractonic systems [13-16]. A

Published by the American Physical Society under the terms of the Creative Commons Attribution 4.0 International license. Further distribution of this work must maintain attribution to the author(s) and the published article's title, journal citation, and DOI. Open access publication funded by the Max Planck Society. particularly generic mechanism for nonergodic behavior is hosted in lattice gauge theories (LGTs) where local constraints emerge naturally due to the local gauge symmetry, leading to an extensive number of local conserved quantities. Specifically, this can lead to the absence of ergodicity in 1D LGTs with discrete [17-19] and continuous [20] gauge symmetries or for higher-dimensional systems in the low-energy limit [21] or when they are noninteracting [22]. However, it has remained a key challenge to identify nonergodic behavior in genuinely interacting quantum systems beyond one spatial dimension.

In this work we show that the $2 \mathrm{D} \mathrm{U}(1)$ quantum link model (QLM) features both localized and ergodic phases in the absence of disorder. This result appears especially remarkable since for the more conventional disorderinduced many-body localization $[23,24]$ it is debated whether interactions destroy localization in $2 \mathrm{D}$, as it follows from the theoretical arguments [25,26], or not, as suggested by experiment [27] and numerics [28]. Thus, we show that the constraint-induced disorder-free localization provides an alternative and robust localization mechanism surviving in the higher dimensions in the presence of interactions. Our numerical argument relies on a mapping onto a classical correlated percolation problem providing a bound on the localization transition 
of the quantum model. We identify a distinguishing quantum dynamical signature of the two phases by studying the propagation of an initial line defect, which leads to two different light cone structures. For the challenging problem of the nonequilibrium quantum dynamics of the interacting two-dimensional system, we introduce the method of variational classical networks (VCNs), which provide an efficient and perturbatively controlled representation of the quantum many-body wave function in terms of a network of classical spins akin to artificial neural networks (ANNs). The introduced methods can be applied to any LGT with finite local Hilbert spaces irrespective of dimensionality.

Quantum link model.-We study the 2D U(1) quantum link model [29,30], which has been introduced as a descendant of lattice quantum electrodynamics with spin-1/2 gauge degrees of freedom. In the QLM the spins $S_{\mathbf{r}, \mu}$ reside on the links of a square lattice connecting vertices $\mathbf{r}=(x, y)$ and $\mathbf{r}+\mu$ [here $\mu=\hat{i}, \hat{j}$ is one of the two unit vectors of the lattice, Fig. 1(a)], with the Hamiltonian:

$H=H_{0}+V \equiv \lambda \sum_{\square}\left(U_{\square}+U_{\square}^{\dagger}\right)^{2}-J \sum_{\square}\left(U_{\square}+U_{\square}^{\dagger}\right)$.

The sums run over all plaquettes $\square, \quad U_{\square}=$ $S_{\mathbf{r}, \hat{i}}^{+} S_{\mathbf{r}+\hat{i}, \hat{j}}^{+} S_{\mathbf{r}+\hat{j}, \hat{i}}^{-} S_{\mathbf{r}, \hat{j}}^{-}$induces a collective flip of all spins on plaquette $\square$, and $S_{\mathbf{r}, \hat{\mu}}^{ \pm}$denote the raising and lowering operators. The first (potential) term counts the number of flippable plaquettes and the second (kinetic) term induces coherent dynamics. For what follows, we will consider periodic boundary conditions and the case of a strong potential term with $J / \lambda=-0.1$. The QLM not only appears in the context of high-energy physics, but also shares strong connections to condensed matter systems featuring quantum spin ice phases [31,32] or quantum dimer models $[1,33]$. On the experimental side various proposals have explored the potential realization of the QLM in quantum simulators within the past years [34,35].

The local gauge symmetry of the QLM is generated by the operators $G_{\mathbf{r}}=\sum_{\mu}\left(S_{\mathbf{r}, \mu}^{z}-S_{\mathbf{r}-\mu, \mu}^{z}\right)$ counting the total inflow of the electric field to the vertex $\mathbf{r}$. Since $\left[G_{\mathbf{r}}, H\right]=0$ for all lattice points and $\left[G_{\mathbf{r}}, G_{\mathbf{r}^{\prime}}\right]=0$, eigenstates of $H$ can be classified by the respective eigenvalues $q_{\mathbf{r}} \in$ $\{-2,-1,0,1,2\}$ of $G_{\mathbf{r}}$. The set of $\mathbf{q}=\left\{q_{\mathbf{r}}\right\}$ defines the so-called superselection sector of states $\left|\psi_{\mathbf{q}}\right\rangle$ with $G_{\mathbf{r}}\left|\psi_{\mathbf{q}}\right\rangle=q_{\mathbf{r}}\left|\psi_{\mathbf{q}}\right\rangle$, so that each of the $q_{\mathbf{r}}$ can be given a physical meaning in terms of static background charges located at $\mathbf{r}$ [20]. The QLM further has global conserved quantities $\Phi_{x}=\sum_{y} S_{\mathbf{r}, \hat{i}}^{z}, \Phi_{y}=\sum_{x} S_{\mathbf{r}, \hat{j}}^{z}$, which define the flux sectors.

Disorder-free localization.-The existence of these sectors, protected by gauge invariance, can lead to an unconventional scenario for ergodicity breaking. Consider a homogeneous superposition state $|\psi\rangle=\sum_{\mathbf{q}} C_{\mathbf{q}}\left|\psi_{\mathbf{q}}\right\rangle$
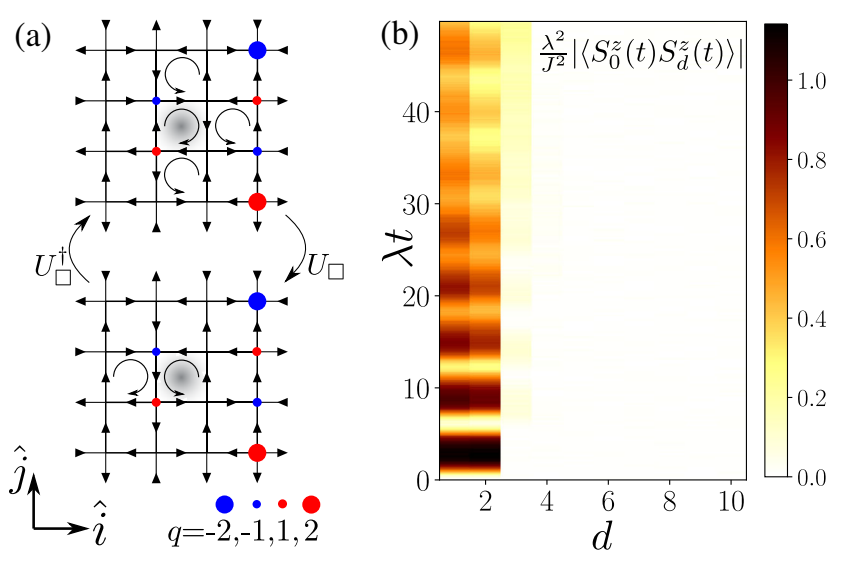

FIG. 1. (a) Illustration of the U(1) quantum link model (QLM) with spin-1/2's located on the links of the square lattice. Spins pointing $\rightarrow$ or $\uparrow$ correspond to $S^{z}=+1$ and $\leftarrow$ or $\downarrow$ to $S^{z}=-1$, respectively. Kinetics is introduced by plaquette-flip operators $U_{\square}, U_{\square}^{\dagger}$ (shown for the darkened central plaquette) whenever the spins on a plaquette are oriented clockwise or counterclockwise. Flippable plaquettes are denoted by circular arrows. Background charges with nonzero inflow or outflow of electric field at a given vertex are indicated by red a blue dots. (b) Spatioemporal buildup of quantum correlations $\left|\left\langle S_{\mathbf{r}, y}^{z}(t) S_{\mathbf{r}+\mathbf{d}, y}^{z}(t)\right\rangle\right| \equiv$ $\left|\left\langle S_{0}^{z}(t) S_{d}^{z}(t)\right\rangle\right|(\mathbf{d}=d \hat{i})$ starting from $|\psi(\alpha=0)\rangle=|\rightarrow\rangle$ in the localized phase of the QLM for $J / \lambda=-0.1$ and a system of size $80 \times 80$, i.e., 12800 spins.

involving many superselection sectors. As the Hamiltonian and typical observables are block diagonal, i.e., $H\left|\psi_{\mathbf{q}}\right\rangle=H_{\mathbf{q}}\left|\psi_{\mathbf{q}}\right\rangle$, the expectation values of an operator $O$ during dynamics become equivalent to $\langle O(t)\rangle=$ $\sum_{\mathbf{q}}\left|C_{\mathbf{q}}\right|^{2}\left\langle\psi_{\mathbf{q}}\left|e^{i H_{\mathbf{q}} t} O e^{-i H_{\mathbf{q}} t}\right| \psi_{\mathbf{q}}\right\rangle$ resembling an effective disorder average with the disorder strength determined by the random background charges in the typical superselection sectors [20]. This can, in principle, lead to nonergodic behavior of $\langle O(t)\rangle$, although both the initial state and the Hamiltonian are homogeneous leading to the notion of disorder-free localization [17].

Initial states for time evolution.-We now aim to characterize the nonequilibrium dynamics of the QLM for the following homogeneous initial states $\left|\psi_{0}\right\rangle$.

(i) $\left|\psi_{0}\right\rangle=|\rightarrow\rangle=\bigotimes_{i}(1 / \sqrt{2})\left(\left|\uparrow_{i}\right\rangle+\left|\downarrow_{i}\right\rangle\right)$, where $\left|\uparrow_{i}\right\rangle$ and $\left|\downarrow_{i}\right\rangle$ are the two basis states at link $i$. This state is distributed over all superselection sectors of the model.

(ii) $\left|\psi_{0}\right\rangle=|\rightarrow\rangle_{\mathrm{FF}}$ which is a projection of $|\rightarrow\rangle$ to a single "fully flippable" (FF) sector, defined as the zerocharge zero-flux sector. $|\rightarrow\rangle_{\mathrm{FF}}$ is an equal-weighted superposition of all states from the FF sector (i.e., the Rokhsar-Kivelson state [1] for the FF sector).

While $|\rightarrow\rangle$ is a product state, $|\rightarrow\rangle_{\mathrm{FF}}$ is entangled. Nevertheless, $|\rightarrow\rangle$ can be continuously connected to a product state from the FF sector via

$$
\left|\psi_{0}(\alpha)\right\rangle=\bigotimes_{i}\left[\sin \left(\alpha+\frac{\pi}{4}\right)\left|\mathrm{FF}_{i}\right\rangle+\cos \left(\alpha+\frac{\pi}{4}\right)\left|\overline{F F}_{i}\right\rangle\right],
$$


with $\alpha \in[0, \pi / 4]$. Here, $\left|\mathrm{FF}_{i}\right\rangle$ and $\left|\overline{\mathrm{FF}}_{i}\right\rangle$ denote the local spin orientations of the two states with all plaquettes flippable and therefore with checkerboardalternating clockwise $\left(\mathrm{FF}_{i}\right)$ and anticlockwise $\left(\overline{\mathrm{FF}}_{i}\right)$ orientations. By construction, $|\psi(\alpha=0)\rangle=|\rightarrow\rangle$ and $|\psi(\alpha=\pi / 4)\rangle=|\mathrm{FF}\rangle \equiv \bigotimes_{i}\left|\mathrm{FF}_{i}\right\rangle$. Importantly, the states $|\psi(\alpha)\rangle$ are spatially uniform. The resulting dynamics for $\alpha=0$ is displayed in Fig. 1(b), where we monitor the spatiotemporal buildup of quantum correlations. We will identify the limited spatial propagation with nonergodic behavior below.

Variational classical networks.-Calculating the dynamics of interacting quantum systems in $2 \mathrm{D}$ is an inherently hard problem without a general-purpose computational method available to date. Representing a generic quantum many-body state as $|\psi\rangle=\sum_{\vec{s}} \psi(\vec{s})|\vec{s}\rangle$ requires, in principle, the storage of exponentially many amplitudes $\psi(\vec{s})$ [here $\left.\vec{s}=\left(s_{1}, \ldots, s_{N}\right)\right]$. Recently, it has been proposed to use networks of classical spins to solve this problem $[36,37]$ by avoiding to store the $\psi(\vec{s})$ 's. The amplitudes $\psi(\vec{s}) \approx \exp [\mathcal{H}(\vec{s}, \mathcal{W})]$ are rather generated on the fly when needed via a complex classical spin model with Hamiltonian $\mathcal{H}(\vec{s}, \mathcal{W})$ determined by a set of couplings $\mathcal{W}$ between the involved spins. Here, we construct $\mathcal{H}(\vec{s}, \mathcal{W})$ using a perturbatively controlled expansion and extend the recently proposed classical networks [37] upon imposing an additional optimization principle. The resulting approach can be interpreted as encoding $|\psi\rangle$ in an ANN with a specific simplified network structure.

Within the VCNs we perform an expansion around a classical limit, which in the case of the QLM is the potential term $H_{0}$ in Eq. (1). By representing the evolution operator in the interaction picture $W(t)=\mathcal{T} \exp \left[-\int_{0}^{t} d t^{\prime} V\left(t^{\prime}\right)\right]$, we can write $\psi(\vec{s}, t)=\left\langle\vec{s}\left|e^{i H t}\right| \psi_{0}\right\rangle=\left\langle\vec{s}\left|e^{i H_{0} t} W(t)\right| \psi_{0}\right\rangle=$ $e^{i E_{\vec{s}} t}\left\langle\vec{s}|W(t)| \psi_{0}\right\rangle$, where $H_{0}|\vec{s}\rangle=E_{\vec{s}}|\vec{s}\rangle$. For the remaining term $\left\langle\vec{s}|W(t)| \psi_{0}\right\rangle$ we perform a cumulant expansion for time-ordered exponential operators [37-39], which, e.g., to the first order yields $\mathcal{H}=-i E_{\vec{s}} t-\left\langle\vec{s}\left|\int_{0}^{t} d t^{\prime} V\left(t^{\prime}\right)\right| \psi_{0}\right\rangle /$ $\left\langle\vec{s} \mid \psi_{0}\right\rangle$. Taking $\left|\psi_{0}\right\rangle=|\rightarrow\rangle$ one obtains for the QLM $\mathcal{H}(\vec{s}, t)=-i E_{\vec{s}} t-i J \sum_{\square}^{\prime} \int_{0}^{t} d t^{\prime} e^{i \lambda \omega_{\square}(\vec{s}) t^{\prime}}$. Here $\sum_{\square}^{\prime}$ denotes the sum over all flippable plaquettes in the spin configuration $\vec{s}$, and $\omega_{\square}=-4, \ldots, 4$ counts the difference between number of flippable plaquettes surrounding the given $\square$ before and after its flip (i.e., $\lambda \omega_{\square}$ gives the potential energy difference before and after the flip). For example, for the configuration in Fig. 1(a) we have $\omega_{\square}=3-1=2$ for the central plaquette. Going beyond previous work [37] we promote $\lambda t$ and its functions such as $\int_{0}^{t} d t^{\prime} e^{i \lambda \omega_{\square} t^{\prime}}$ to variational parameters $\mathcal{W}_{k}(t)=\left(\mathcal{W}^{(0)}, \mathcal{W}_{-4}^{(1)}, \ldots, \mathcal{W}_{4}^{(1)}\right)$ yielding $\mathcal{H}\left(\vec{s}, \mathcal{W}_{k}(t)\right)=-i E_{\vec{s}} \mathcal{W}^{(0)}-i J \sum_{\square}^{\prime} \mathcal{W}_{\omega_{\square}(\vec{s})}^{(1)}$. The local connectivity of the $\mathrm{VCN}$ is encoded in the function $\omega_{\square}(\vec{s})$. For the actual shown numerical simulations we use a second-order ansatz and more complex initial states (see Refs. [39,40]). The $\mathcal{W}_{k}(t)$ 's are determined by a time-dependent variational principle translating quantum dynamics into a system of coupled classical differential equations $\sum_{k^{\prime}} \mathcal{S}_{k, k^{\prime}} \dot{\mathcal{W}}_{k^{\prime}}=-i F_{k}$ in the space of variational parameters. Here, $\mathcal{S}_{k, k^{\prime}}=\left\langle O_{k}^{*} O_{k^{\prime}}\right\rangle-\left\langle O_{k}^{*}\right\rangle\left\langle O_{k^{\prime}}\right\rangle$ and $\quad F_{k}=\left\langle E_{\mathrm{loc}} O_{k}^{*}\right\rangle-\left\langle E_{\mathrm{loc}}\right\rangle\left\langle O_{k}^{*}\right\rangle$, with $O_{k}(\vec{s})=$ $\partial \ln \psi(\vec{s}, \mathcal{W}) / \partial \mathcal{W}_{k}$ and $E_{\mathrm{loc}}(\vec{s})=\left\langle\vec{s}|H| \psi_{\mathcal{W}}\right\rangle /\left\langle\vec{s} \mid \psi_{\mathcal{W}}\right\rangle$ (see Ref. [39]). We solve these equations using a fourth-order Runge-Kutta integrator with step size $\Delta t=0.1 \lambda^{-1}$ and sample the observables using Metropolis Monte Carlo (MC) method with $10^{6}$ sweeps at each time instance, with single spin-flip updates for $|\rightarrow\rangle$ and plaquette flips for $|\rightarrow\rangle_{\mathrm{FF}}$.

While our approach is numerically stable and therefore does not face some challenges appearing in ANNs [41], it has its own limitations due to its perturbative construction, which is guaranteed to work only up to times $t \simeq|1 / J|$. We find, however, that the errors remain perturbatively controlled up to much longer times as a consequence of the variational optimization. This can be verified, since the method provides a self-contained way of tracking the error, not referring to any reference solution. We present the details of the error analysis in Refs. $[39,40]$ together with benchmarks and the discussion of the limitations of the method.

Localized and ergodic dynamics.-Using the VCNs we now compute nonequilibrium dynamics in the QLM. We start by studying the spatiotemporal buildup of quantum correlations, measured via $\left\langle S_{0}^{z}(t) S_{d}^{z}(t)\right\rangle$, upon initializing the system in state $\left|\psi_{0}\right\rangle=|\rightarrow\rangle$. The result is shown in Fig. 1(b), where one can see that correlations emerge only over a limited spatial distance suggesting nonergodic behavior. We proceed by further corroborating this observation by other measures.

Namely, we study energy transport in the QLM by creating initial conditions with a spatial energy inhomogeneity in the form of a line defect with subextensive energy contribution and use the character of energy propagation to distinguish between ergodic and localized dynamics. Concretely, we consider the two initial conditions $\left|\psi_{0}\right\rangle=|\rightarrow\rangle$ or $|\rightarrow\rangle_{\mathrm{FF}}$ upon applying in addition $P=\prod_{\square \in \mathcal{C}_{0}}\left[1+\left(U_{\square}+U_{\square}^{\dagger}\right)^{2}\right]$ along all plaquettes in column $d=0$; here $\mathcal{C}_{d}$ denotes the set of plaquettes in column $d$. In Fig. 2 we plot the (normalized) column energy $\varepsilon_{d}(t)=\left(\left\langle H_{d}(t)\right\rangle-H_{\mathrm{av}}\right) / H_{\mathrm{av}}, \quad$ with $\quad H_{d}=\sum_{\square \in \mathcal{C}_{d}} H_{\square}$ the total energy for the plaquettes in $d$ th column, $H_{\square}=\lambda\left(U_{\square}+U_{\square}^{\dagger}\right)^{2}-J\left(U_{\square}+U_{\square}^{\dagger}\right)$. Further, $H_{\mathrm{av}}=\langle H\rangle / L$ denotes the expected $\left\langle H_{d}(t)\right\rangle$ in the long-time limit when the system is thermalizing ( $L$ is the number of columns). We have checked that other choices of the excitation operator $P$ (diagonal or nondiagonal) increasing the amplitudes of configurations with flippable plaquettes at $d=0$ do not qualitatively affect the results we present next.

Comparing Figs. 2(a) and 2(c) we observe that the dynamics differs qualitatively for the two initial conditions, 

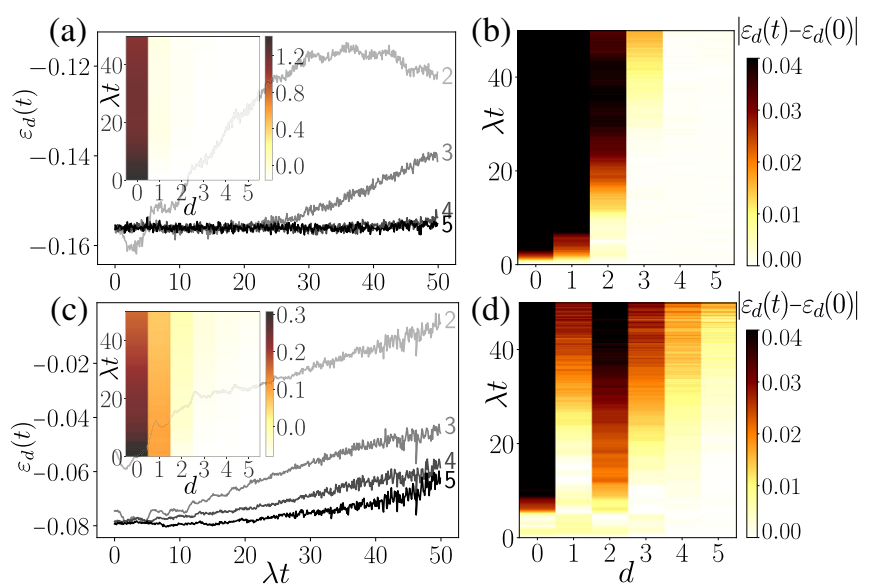

FIG. 2. Quantum energy dynamics for line defects created in the $|\rightarrow\rangle$ (upper panel) and $|\rightarrow\rangle_{\mathrm{FF}}$ (lower panel) initial states, displaying the normalized plaquette energy of $d$ th column $\varepsilon_{d}(t)=\left[\left\langle H_{d}(t)\right\rangle-H_{\mathrm{av}}\right] / H_{\mathrm{av}}$ [here $\left.H_{\mathrm{av}}=\langle H\rangle / L\right]$. (a),(c) $\varepsilon_{d}(t)$ for different columns $d=2-5$, where darker colors refer to larger distances from the initial defect. Insets show the same data including $d=0,1$ in a color plot. (b),(d) Absolute deviation of $\varepsilon_{d}(t)$ from the initial value $\varepsilon_{d}(0)$. (b) Signal propagation for $|\rightarrow\rangle$ showing a strong bending of the light cone indicating localized behavior and (d) for $|\rightarrow\rangle_{\mathrm{FF}}$ consistent with linear propagation indicative of ergodic behavior. For all the plots $J / \lambda=-0.1$ and system size $10 \times 10$.

although the Hamiltonian parameters are identical. While for $\left|\psi_{0}\right\rangle=|\rightarrow\rangle$ energy transport is highly suppressed and only visible on short distances [Fig. 2(a)], the opposite happens for $|\rightarrow\rangle_{\mathrm{FF}}$. This becomes even more apparent in Figs. 2(b) and 2(d), where $\varepsilon_{d}(t)$ relative to the initial value $\varepsilon_{d}(0)$ is shown, therefore more directly highlighting energy propagation. While for $|\rightarrow\rangle_{\mathrm{FF}}$ we identify a linearly propagating front, for $|\rightarrow\rangle$ we observe a strong bending. We argue below that this front for $|\rightarrow\rangle$ can extend only to a finite region as a consequence of disorder-free localization.

Bound on quantum dynamics by unconventional percolation.-The qualitative difference in the quantum dynamics for the initial states $|\rightarrow\rangle$ and $|\rightarrow\rangle_{\mathrm{FF}}$ originates from a dynamical transition, which one can study systematically upon tuning the parameter $\alpha$ for the initial state (2). For this purpose, we employ an unconventional correlated classical percolation problem and establish a bound on the quantum localized-ergodic transition in the QLM providing a strong numerical evidence for an extended nonergodic phase as a consequence of disorder-free localization.

We illustrate the idea for the initial state $|\rightarrow\rangle$, distributed over all superselection sectors. Consider a typical (random) sector from this distribution [Fig. 3(a)]. Such sector exhibits many background charges $q_{\mathbf{r}}$ whenever the "two-in twoout" rule at vertex $\mathbf{r}$ is violated. Importantly, these background charges (constants of motion by gauge invariance) impose strong kinetic constraints. For instance, $q_{\mathbf{r}}= \pm 2$ implies that neighboring spins all point either inward or outward; hence the adjacent plaquettes remain unflippable forever. The influence of $q_{\mathbf{r}}= \pm 1$ charges is more subtle. They make at least two adjacent plaquettes unflippable, while their positions might change over time.

The question we address now is whether these constraints are so strong to fragment the square lattice into sets of kinetically disconnected islands or whether one can contain an extensive (percolating) connected cluster. For that purpose we study an unconventional percolation problem using an infinite-temperature classical MC simulation. We start from the initial condition (2), sampling a random basis state (and thus a sector) with a distribution set by the amplitudes in $|\psi(\alpha)\rangle$. Then we determine which parts of the systems are kinetically connected, using MC search with random plaquette flips. The simulation is stopped when every plaquette is flipped either 0 or more than some fixed threshold $(=100)$ number of times (or after $10^{11} \mathrm{MC}$ steps if this condition is still not satisfied). As a result we find the number of performed flips for each plaquette [Fig. 3(b)]. Repeating this procedure for different initial configurations at a given $\alpha$ and scanning $\alpha$, we finally obtain the percolation probability [Fig. 3(c)]. Most importantly, one can observe a clear evidence for a percolation threshold $\alpha_{c} \approx 0.25$. Although the simulation termination condition is chosen such as to minimize the number of potentially missed "weak connections" between flippable clusters, we cannot exclude the possibility of such misses. While we do not expect a significant impact deep in the respective phases, this caveat might become important in the vicinity of $\alpha_{c}$; thus we restrain ourselves from studying the critical behavior.

Since $\alpha_{c}>0$, the initial state $|\psi(\alpha=0)\rangle=|\rightarrow\rangle$ corresponds to the classically nonpercolating side of the transition, while from $\alpha_{c}<\pi / 4$ it follows that state $|\psi(\alpha=\pi / 4)\rangle=|\mathrm{FF}\rangle$ and all other states from the FF sector (including $|\rightarrow\rangle_{\mathrm{FF}}$ ) lie on the percolating side. This classical threshold is imprinted in the quantum dynamics and ultimately leads to the strong localization observed in propagation of correlations [Fig. 1(b)] and of the energy [Figs. 2(a) and 2(b)] for $|\rightarrow\rangle$. For the FF-sector state $|\rightarrow\rangle_{\mathrm{FF}}$ there is no percolation constraint, which allows propagation of the signal to long distances [Figs. 2(c) and 2(d)]. We emphasize that this analysis sets only a lower bound onto the true quantum transition localization-delocalization threshold $\alpha_{c}^{(q)}$, since the quantum system might still be localized due to interference caused by the kinetic $J$ term even on the classically percolating side.

Summary and outlook.-We have shown that genuinely interacting 2D homogeneous LGTs can become nonergodic as a consequence of disorder-free localization. This is especially surprising since the conventional many-body localization is theoretically conjectured to be unstable in 2D at elevated energy densities [25,26], implying that gauge invariance (constraints) represent a different and probably more robust mechanism of ergodicity breaking as 


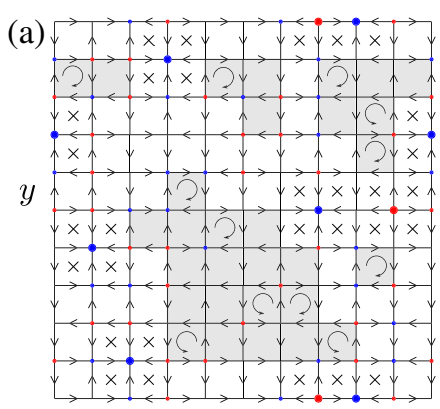

$x$
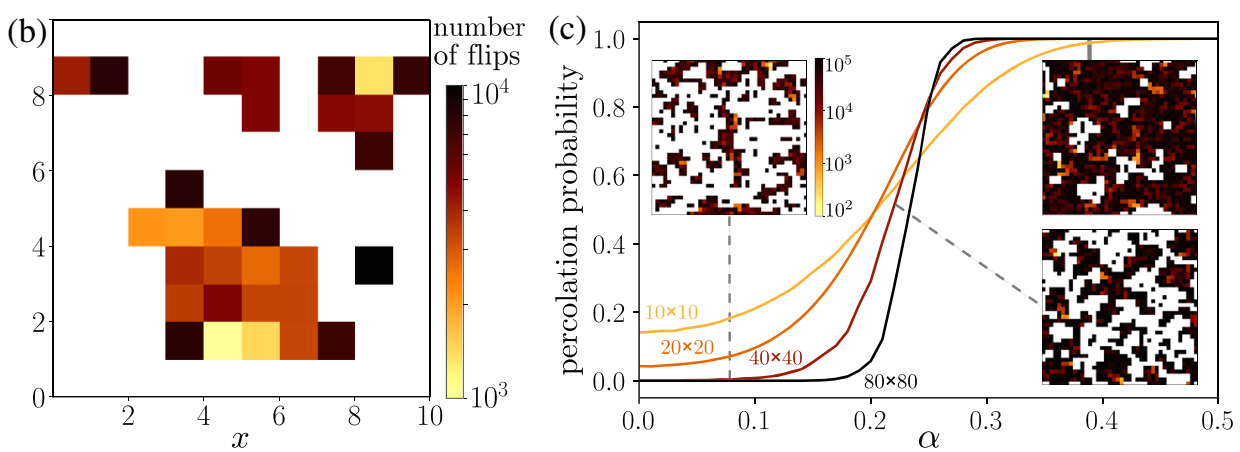

FIG. 3. Classical correlated percolation problem implying a quantum nonergodic phase in the QLM. (a) Typical spin configuration sampled from $|\rightarrow\rangle$ with arrows showing the local spin orientation and circular arrows showing flippable plaquettes. Red and blue dots denote positive and negative background charges, respectively. Crosses indicate plaquettes blocked by charges $q= \pm 2$. Gray color indicates plaquettes that can become flippable in the course of the evolution. (b) Result of the Monte Carlo simulation starting from the state (a). The color map shows the number of times that the individual plaquettes were flipped in the course of the simulation; white color stands only for plaquettes that have never been flipped. (c) Percolation probability versus $\alpha$. The insets show typical configurations below, at, and above the percolation threshold $\alpha_{c} \approx 0.25$ for a $40 \times 40$ system.

compared to disorder. The key element of our analysis is a bound on the localization-delocalization transition based on a classical correlated percolation problem implying a strong fragmentation of Hilbert space into kinetically disconnected regions. Both the percolation analysis as well as the introduced variational classical networks can be directly applied to other quantum many-body systems with finitedimensional local Hilbert spaces independent of dimensionality, such as 3D quantum spin ice systems, which might be an interesting scope of the developed techniques in the future. Further, it might be interesting to explore how the classical and quantum transition thresholds are related to each other as well as to determine their respective critical behaviors, and whether the disorderfree localization scenario holds also in the presence of matter degrees of freedom. Our theoretical analysis appears within reach of future experiments: significant efforts in the past years have explored routes to realize the QLM model experimentally in systems of Rydberg atoms $[34,35]$ as a next step after the recent experimental advances on 1D LGTs [42-46].

We are grateful to H. Burau, J. Chalker, M. Dalmonte, R. Moessner, and G.-Y. Zhu for helpful discussions. P. K. acknowledges the support of the Alexander von Humboldt Foundation and the Ministry of Science and Higher Education of the Russian Federation (NUST MISiS Grant No. K2-2020-038). This project has received funding from the European Research Council (ERC) under the European Union's Horizon 2020 research and innovation programme (Grant Agreement No. 853443), and M.H. further acknowledges support by the Deutsche Forschungsgemeinschaft via the Gottfried Wilhelm Leibniz Prize program. Y.-P.H. receives funding from the European Union's Horizon 2020 research and innovation programme under the Marie Skłodowska-Curie Grant
Agreement No. 701647. M. S. was supported through the Leopoldina Fellowship Programme of the German National Academy of Sciences Leopoldina (LPDS 2018-07) with additional support from the Simons Foundation. The numerical calculations were carried out using Max Planck Computing and Data Facility (Garching).

*karpov.petr@gmail.com

[1] D. S. Rokhsar and S. A. Kivelson, Phys. Rev. Lett. 61, 2376 (1988).

[2] S. Sachdev, Rep. Prog. Phys. 82, 014001 (2019).

[3] M. J. P. Gingras and P. A. McClarty, Rep. Prog. Phys. 77, 056501 (2014).

[4] P. A. McClarty, M. Haque, A. Sen, and J. Richter, Phys. Rev. B 102, 224303 (2020).

[5] M. Stern, C. Castelnovo, R. Moessner, V. Oganesyan, and S. Gopalakrishnan, arXiv:1911.05742.

[6] A. Y. Kitaev, Ann. Phys. (Amsterdam) 303, 2 (2003).

[7] H. J. Rothe, Lattice Gauge Theories: An Introduction, 3rd ed. (World Scientific, Singapore, 2005).

[8] C. J. Turner, A. A. Michailidis, D. A. Abanin, M. Serbyn, and Z. Papic, Nat. Phys. 14, 745 (2018).

[9] C.-J. Lin and O. I. Motrunich, Phys. Rev. Lett. 122, 173401 (2019).

[10] M. van Horssen, E. Levi, and J. P. Garrahan, Phys. Rev. B 92, 100305(R) (2015).

[11] N. Pancotti, G. Giudice, J. I. Cirac, J. P. Garrahan, and M. C. Banuls, Phys. Rev. X 10, 021051 (2020).

[12] Z. Lan, M. van Horssen, S. Powell, and J. P. Garrahan, Phys. Rev. Lett. 121, 040603 (2018).

[13] P. Sala, T. Rakovszky, R. Verresen, M. Knap, and F. Pollmann, Phys. Rev. X 10, 011047 (2020).

[14] S. Pai and M. Pretko, Phys. Rev. Lett. 123, 136401 (2019).

[15] S. Pai, M. Pretko, and R. M. Nandkishore, Phys. Rev. X 9 , 021003 (2019).

[16] V. Khemani and R. Nandkishore, Phys. Rev. B 101, 174204 (2020). 
[17] A. Smith, J. Knolle, D. L. Kovrizhin, and R. Moessner, Phys. Rev. Lett. 118, 266601 (2017).

[18] A. Smith, J. Knolle, R. Moessner, and D. L. Kovrizhin, Phys. Rev. Lett. 119, 176601 (2017).

[19] A. Russomanno, S. Notarnicola, F. M. Surace, R. Fazio, M. Dalmonte, and M. Heyl, Phys. Rev. Research 2, 012003(R) (2020).

[20] M. Brenes, M. Dalmonte, M. Heyl, and A. Scardicchio, Phys. Rev. Lett. 120, 030601 (2018).

[21] I. H. Kim and J. Haah, Phys. Rev. Lett. 116, 027202 (2016).

[22] A. Smith, J. Knolle, R. Moessner, and D. L. Kovrizhin, Phys. Rev. B 97, 245137 (2018).

[23] R. Nandkishore and D. A. Huse, Annu. Rev. Condens. Matter Phys. 6, 15 (2015).

[24] D. A. Abanin, E. Altman, I. Bloch, and M. Serbyn, Nat. Phys. 91, 021001 (2019).

[25] W. De Roeck and F. Huveneers, Phys. Rev. B 95, 155129 (2017).

[26] W. De Roeck and J. Z. Imbrie, Phil. Trans. R. Soc. A 375, 20160422 (2017).

[27] J. Choi, S. Hild, J. Zeiher, P. Schauß, A. Rubio-Abadal, T. Yefsah, V. Khemani, D. A. Huse, I. Bloch, and C. Gross, Science 352, 1547 (2016).

[28] T. B. Wahl, A. Pal, and S. H. Simon, Nat. Phys. 15, 164 (2019).

[29] U.-J. Wiese, Ann. Phys. (Berlin) 525, 777 (2013).

[30] S. Chandrasekharan and U.-J. Wiese, Nucl. Phys. B492, 455 (1997).

[31] N. Shannon, G. Misguich, and K. Penc, Phys. Rev. B 69, 220403(R) (2004).

[32] M. Hermele, M. P. A. Fisher, and L. Balents, Phys. Rev. B 69, 064404 (2004).
[33] D. Banerjee, F.-J. Jiang, P. Widmer, and U.-J. Wiese, J. Stat. Mech. (2013) P12010.

[34] A. Celi, B. Vermersch, O. Viyuela, H. Pichler, M. D. Lukin, and P. Zoller, Phys. Rev. X 10, 021057 (2020).

[35] A. W. Glaetzle, M. Dalmonte, R. Nath, I. Rousochatzakis, R. Moessner, and P. Zoller, Phys. Rev. X 4, 041037 (2014).

[36] G. Carleo and M. Troyer, Science 355, 602 (2017).

[37] M. Schmitt and M. Heyl, SciPost Phys. 4, 013 (2018).

[38] R. Kubo, J. Phys. Soc. Jpn. 17, 1100 (1962).

[39] See Supplemental Material at http://link.aps.org/supplemental/ 10.1103/PhysRevLett.126.130401 for the details on the construction of VCN Ansätze, description of time-dependent variational principle, and benchmarks of $\mathrm{VCN}$ versus exact diagonalization.

[40] R. Verdel, M. Schmitt, Y.-P. Huang, P. Karpov, and M. Heyl, Phys. Rev. B 103, 165103 (2021).

[41] M. Schmitt and M. Heyl, Phys. Rev. Lett. 125, 100503 (2020).

[42] E. Martinez, C. Muschik, P. Schindler, D. Nigg, A. Erhard, M. Heyl, P. Hauke, M. Dalmonte, T. Monz, P. Zoller, and R. Blatt, Nature (London) 534, 516 (2016).

[43] C. Kokail, C. Maier, R. van Bijnen, T. Brydges, M. K. Joshi, P. Jurcevic, C. A. Muschik, P. Silvi, R. Blatt, C. F. Roos et al., Nature (London) 569, 355 (2019).

[44] F. Görg, K. Sandholzer, J. Minguzzi, R. Desbuquois, M. Messer, and T. Esslinger, Nat. Phys. 15, 1161 (2019).

[45] C. Schweizer, F. Grusdt, M. Berngruber, L. Barbiero, E. Demler, N. Goldman, I. Bloch, and M. Aidelsburger, Nat. Phys. 15, 1168 (2019).

[46] A. Mil, T. V. Zache, A. Hegde, A. Xia, R. P. Bhatt, M. K. Oberthaler, P. Hauke, J. Berges, and F. Jendrzejewski, Science 367, 1128 (2020). 